\title{
The singular ideal and the socle of incidence rings
}

\author{
Müge Kanuni*1 ${ }^{*}$, Özkay Özkan² ${ }^{(1)}$ \\ ${ }^{1}$ Department of Mathematics, Düzce University, Konuralp 81620 Düzce, Turkey. \\ ${ }^{2}$ Department of Mathematics, Gebze Technical University, 41400 Gebze, Kocaeli, Turkey
}

\begin{abstract}
Let $R$ be a ring with identity and $I(X, R)$ be the incidence ring of a locally finite partially ordered set $X$ over $R$. In this paper, we compute the socle and the singular ideal of the incidence ring for some $X$ in terms of the socle of $R$ and the singular ideal of $R$, respectively.
\end{abstract}

Mathematics Subject Classification (2010). 16D25

Keywords. incidence ring, socle, singular ideal

\section{Introduction and preliminaries}

The investigation of a ring or algebra $R$ is usually enriched by understanding special types of ideals or subspaces of $R$ such as the Jacobson radical, the prime radical, the socle, the singular ideal, the center, etc. Although incidence rings have been an object of study for a few decades, there does not seem to be any results in the literature on the socle of incidence rings.

In this paper, we will be computing the left socle and the left singular ideal of an incidence ring $I(X, R)$, however similar statements hold for right analogues by replacing $\operatorname{Min}(X)$ by $\operatorname{Max}(X)$ and the upper finiteness by the lower finiteness condition.

The outline of the paper is as follows: we give preliminaries and easily computable observations in the Introduction. In Section 2, we look at the singular ideal of particular incidence rings. Theorem 2.4 states that for any locally finite partially ordered set $X$ and any ring $R, F S\left(I\left(X, \operatorname{Sing}_{l}(R)\right)\right) \subseteq \operatorname{Sing}_{l}(I(X, R)) \subseteq I\left(X, \operatorname{Sing}_{l}(R)\right)$.

Proposition 2.7 states that the left singular ideal of $I(X, R)$ is the incidence subring of $X$ over the left singular ideal of $R$, under the hypothesis that $\operatorname{Min}(X)$ is a maximal antichain and $\kappa(x)$ is finite for any $x \in \operatorname{Min}(X)$. Corollary 2.8 states that if $X$ is finite, then $\operatorname{Sing}_{l}(I(X, R))=I\left(X, \operatorname{Sing}_{l}(R)\right)$.

In Section 3, we look at the socle of incidence rings. Theorem 3.13 restricts the socle of an incidence algebra between the following two sets:

$$
\bigoplus_{x \in \operatorname{Min}(X)} \bigoplus_{A \in \mathfrak{A}} \bigoplus_{f \in S(x, R)} A f \subseteq \operatorname{Soc}_{l}(I(X, R)) \subseteq \bigoplus_{x \in \operatorname{Min}(X)} S\left(x, \operatorname{Soc}_{l}(R)\right),
$$

where $\mathfrak{A}$ is the collection of all minimal left ideals of $R$. Theorem 3.2 states that if $\operatorname{Min}(X)$ is a maximal antichain and $\kappa(x)$ is finite for any $x \in \operatorname{Min}(X)$, then $\operatorname{Soc}_{l}(I(X, R))=$

\footnotetext{
*Corresponding Author.

Email addresses: mugekanuni@duzce.edu.tr (M. Kanuni), ozkayoz@yahoo.com (Ö. Özkan)

Received: 03.02.2020; Accepted: 08.08.2020
} 
$\bigoplus_{x \in \operatorname{Min}(X)} S\left(x, \operatorname{Soc}_{l}(R)\right)$. Theorem 3.15 has the same conclusion when $R$ is a commutative ring with finitely many minimal ideals.

Proposition 3.5 affirms that $\operatorname{Soc}_{l}(I(X, R))=\{0\}$ if $\operatorname{Min}(X)=\emptyset$. Also, Theorem 3.8 states that $\operatorname{Soc}_{l}(I(X, R))=\bigoplus_{x \in \operatorname{Min}(X)} S\left(x, \operatorname{Soc}_{l}(R)\right)$ under the assumption that $R$ is an Artinian and nonsingular ring, $\operatorname{Min}(X)$ is finite and $\kappa(x)$ is finite for all $x \in X$. At the end of the paper, we provide an example of an incidence ring with non-equal left and right socles. We also state the necessary conditions for the left and right socles of $I(X, R)$ to be isomorphic in Proposition 3.20.

Throughout the article, $R$ is a ring with unity and not necessarily commutative. We assume $X$ is a locally finite partially ordered set. $\operatorname{Min}(X)$ is the set of all minimal elements of $X$, and $\operatorname{Max}(X)$ is the set of all maximal elements of $X$. $\operatorname{Min}(X), \operatorname{Max}(X)$ may very well be empty sets. For any set $U$, we will use $|U|$ to denote the cardinality of $U$.

The socle $\operatorname{Soc}(M)$ of an $R$-module $M$ is the sum of all its simple (minimal) submodules. If we take $M=R$ as a left $R$-module, then the sum of all minimal left ideals of $R$ is the left socle of $R$, denoted by $\operatorname{Soc}_{l}(R)$. Similarly the right socle of $R, \operatorname{Soc}_{r}(R)$ is defined as the sum of all minimal right ideals of $R$. A left (right) essential ideal $E$ of $R$ is a left (right) ideal which intersects any non-zero left (right) ideal of $R$ non-trivially. It is well-known that the left (right) socle of a ring is equal to the intersection of all left (right) essential ideals.

For any subset $E$ of $R$, let $a n n_{l}(E),\left(a n n_{r}(E)\right)$ denote the left (right) annihilator of $E$ and for any $a \in R, E: a=\{r \in R: r a \in E\}$. If $E$ is an ideal, then so is $E: a$.

A left dense ideal $D$ of a ring $R$ is a left ideal with the property that for any $a \in R$, $\operatorname{ann}_{r}(D: a)=0$.

It is an easy exercise to show that any left (right) dense ideal is a left (right) essential ideal. The intersection of any finite collection of left (right) essential (dense) ideals is always left (right) essential (dense). However, the intersection of all the left (right) essential ideals of the ring $R$ is a left (right) essential ideal if and only if there is a minimal left (right) essential ideal of $R$. Similarly, the intersection of all left (right) dense ideals is a left (right) dense ideal if and only if there is a minimal left (right) dense ideal of $R$.

For any ring $R$,

$$
\operatorname{Sing}_{l}(R)=\left\{x \in R: \operatorname{ann}_{l}(x) \text { is a left essential ideal of } R\right\}
$$

is called the left singular ideal of $R$. A ring $R$ is a left nonsingular ring if $\operatorname{Sing}_{l}(R)=0$. Similar definitions can be given for right singular ideal $\operatorname{Sing}_{r}(R)$ of $R$.

$\operatorname{Soc}_{r}(R), \operatorname{Soc}_{l}(R), \operatorname{Sing}_{l}(R)$ and $\operatorname{Sing}_{r}(R)$ are all two-sided ideals of $R$.

Let $X$ be a partially ordered set. For any $x, z \in X$ with $x \leq z$, the interval $[x, z]$ is defined as

$$
[x, z]=\{y \in X: x \leq y \leq z\} .
$$

If every interval of $X$ is a finite set, then $X$ is called a locally finite partially ordered set.

For a ring $R$ and a locally finite partially ordered set $X$, the incidence ring $I(X, R)$ is the set of functions $f: X \times X \rightarrow R$ such that $f(x, y)=0$ unless $x \leq y$, with the following operations

$$
\begin{aligned}
(f+g)(x, y) & =f(x, y)+g(x, y) \\
f g(x, y) & =\sum_{x \leq z \leq y} f(x, z) g(z, y)
\end{aligned}
$$

for all $f, g \in I(X, R)$ and $x, y \in X$. Also, if $R$ is commutative, $I(X, R)$ becomes an $R$-algebra with the operation:

$$
(r f)(x, y)=r f(x, y)
$$

for any $r \in R$. The element $\delta \in I(X, R)$ which is defined as 


$$
\delta(u, v)=\left\{\begin{array}{cc}
1 & \text { if } u=v \\
0 & \text { otherwise }
\end{array}\right.
$$

is the identity element of $I(X, R)$. For any $(x, y) \in X \times X$ with $x \leq y$, we define $e_{x y} \in I(X, R)$ as

$$
e_{x y}(u, v)=\left\{\begin{array}{cc}
1 & \text { if }(u, v)=(x, y) \\
0 & \text { otherwise }
\end{array} .\right.
$$

Let $\operatorname{supp}(g)=\{(x, y): g(x, y) \neq 0\}$ denote the support of $g \in I(X, R)$. The set

$$
F S(I(X, R))=\{g \in I(X, R):|\operatorname{supp}(g)|<\infty\}
$$

is a subring of $I(X, R)$ which is called the finite support of $I(X, R)$.

For a detailed discussion about incidence rings see [6].

A partially ordered set $X$ is called upper finite if for each $x \in X, U(x):=\{y \in X \mid x \leq y\}$ is finite. Similarly, $X$ is called lower finite if for each $x \in X, L(x):=\{y \in X \mid y \leq x\}$ is finite. For any $x \in X$, define $\kappa(x):=|U(x)|$ and $\lambda(x):=|L(x)|$.

A subset $S$ of $X$ is called an antichain if any two elements in $S$ are incomparable. An antichain $S$ is called a maximal antichain of $X$ if $S$ is not contained in any other antichain. We first state a few easily deducible observations that will be used in the sequel.

Lemma 1.1. Assume $X$ is a locally finite partially ordered set. Then

(i) $\operatorname{Min}(X)$ is a maximal antichain if and only if for each $y \in X$, there exists a minimal element $x \in \operatorname{Min}(X)$ with $x \leq y$.

(ii) If $\operatorname{Min}(X)$ is a maximal antichain and $\kappa(x)$ is finite for all $x \in \operatorname{Min}(X)$, then $X$ is upper finite.

(iii) If $X$ is an antichain, then $\operatorname{Min}(X)=\operatorname{Max}(X)$.

(iv) If $\operatorname{Min}(X)=\operatorname{Max}(X)$ and $X$ is upper finite, then $X$ is an antichain.

Proof. (i) Clearly, $\operatorname{Min}(X)$ is an antichain, as no two minimal elements are comparable. Assume $\operatorname{Min}(X)$ is a maximal antichain. If there is a $y \in X$ with no $x \in \operatorname{Min}(X)$ such that $x \leq y$, then $x$ and $y$ are incomparable, hence $\operatorname{Min}(X) \cup\{y\}$ is an antichain. This contradicts the maximality of $\operatorname{Min}(X)$.

Conversely, assume on the contrary that $\operatorname{Min}(X)$ is not a maximal antichain, hence there exists an antichain $Y$ properly contaning $\operatorname{Min}(X)$. Let $y \in Y \backslash \operatorname{Min}(X)$, so $y$ is incomparable with any $x \in \operatorname{Min}(X)$ which contradicts the hypothesis.

(ii) Since $\operatorname{Min}(X)$ is a maximal antichain, by (i), for each $y \in X$, there exists a minimal element $x \in \operatorname{Min}(X)$ with $x \leq y$. As $\kappa(y) \leq \kappa(x)$, the result follows.

(iii) If $X$ is an antichain, then every element in $X$, is both a maximal and a minimal element. So $\operatorname{Min}(X)=\operatorname{Max}(X)=X$.

(iv) Assume $X$ is not an antichain, then there exist two distinct elements $x, y$ such that $x \leq y$. Since $X$ is upper finite, $U(x)=\{z \in X: x \leq z\}$ is a finite set, say $\left\{y_{0}:=x, y_{1}:=\right.$ $\left.y, \ldots, y_{n}\right\}$. Notice that if $y_{i} \in U(x)$, for $i \neq 0$, then $U\left(y_{i}\right) \subsetneq U(x)$. Then there exists $z \in U(x)(z \neq x)$ which is also in $\operatorname{Max}(X)$. Since $\operatorname{Max}(X)=\operatorname{Min}(X), z \in \operatorname{Min}(X)$ which is a contradiction to $x \leq z$.

Note that the converses of Lemma 1.1 (ii), (iii) are not true. For instance, the partially ordered set $X$ in Example 1.2 is upper finite, but $\operatorname{Min}(X)$ is not a maximal antichain. 
Example 1.2. Let $X=\left\{x_{i}, y_{i}: i \in \mathbb{N}\right\}$ be a partially ordered set with the relations $x_{i} \leq y_{1}, y_{i+1} \leq y_{i}$ for all $i \in \mathbb{N}$. The Hasse diagram of $X$ is

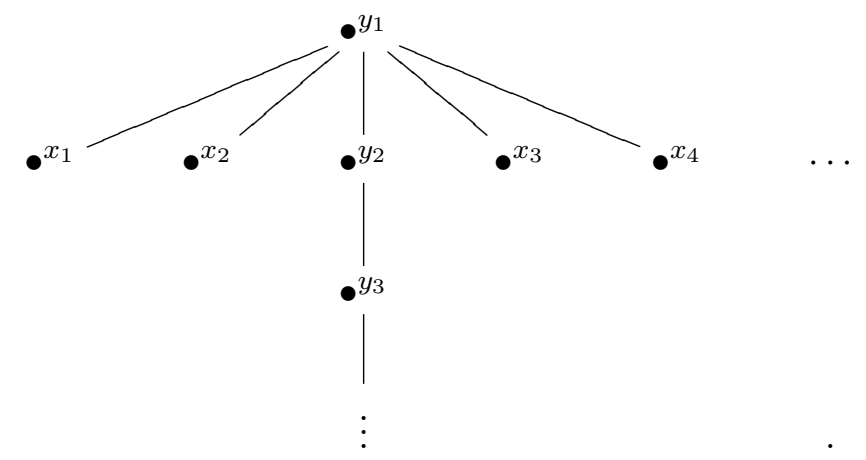

Hence, $\operatorname{Min}(X)=\left\{x_{i}: i \in \mathbb{N}\right\}$ and $\operatorname{Max}(X)=\left\{y_{1}\right\}$. Now, $\operatorname{Min}(X)$ is a non-empty antichain, which is not maximal, since $\operatorname{Min}(X) \cup\left\{y_{i}\right\}$ is a maximal antichain for any $i \geq 2$. Moreover, $\kappa(x)$ is finite for all $x \in X$, that is $X$ is upper finite.

Remark 1.3. When $X$ is finite, for any $y$ in $X$, there is $x$ in $\operatorname{Min}(X)$ with $x \leq y$. By Lemma $1.1(\mathrm{i}), \operatorname{Min}(X)$ is a maximal antichain.

Let $X$ be a locally finite partially ordered set with a subset $Y$ defined as:

$$
Y=\{y \in X: \text { there is a } x \in \operatorname{Min}(X) \text { with } x \leq y\} .
$$

Then, $X=Y \sqcup(X \backslash Y)$ and let $Z=X \backslash Y$. Now, we mention some properties of $X, Y$ and $Z$ in Lemma 1.4 .

Lemma 1.4. Let $X$ be a locally finite partially ordered set, $Y$ and $Z$ be subposets defined as above. Then $X=Y \sqcup Z$, (i.e., $X$ is the disjoint union of $Y$ and $Z$ ).

(i) $\operatorname{Min}(Y)=\operatorname{Min}(X)$ and $\operatorname{Min}(Z)=\emptyset$.

(ii) $\operatorname{Min}(Y)$ is a maximal antichain of $Y$.

(iii) $\operatorname{Min}(Y)$ is a maximal antichain of $X$ if and only if $Z=\emptyset$.

Proof. (i) $\operatorname{Min}(X) \subseteq \operatorname{Min}(Y)$ : Let $\beta \in \operatorname{Min}(X) \subset Y$ and assume that $\beta \notin \operatorname{Min}(Y)$. By definition of the set $Y$, there exists a $\alpha \in \operatorname{Min}(Y)$ with $\alpha \leq \beta$. Since $\alpha \in Y$, there exists $\gamma \in \operatorname{Min}(X)$ with $\gamma \leq \alpha \leq \beta$. This contradicts minimality of $\beta$. Hence $\beta \in \operatorname{Min}(Y)$.

$\operatorname{Min}(Y) \subseteq \operatorname{Min}(X)$ : Let $\alpha \in \operatorname{Min}(Y)$. Since $\operatorname{Min}(Y) \subseteq Y$ and using the result $\operatorname{Min}(X) \subseteq \operatorname{Min}(Y)$, there exists $\beta \in \operatorname{Min}(X)$ with $\beta \leq \alpha$. Since $\beta \in \operatorname{Min}(X)$ it follows that $\beta \in Y$. Hence $\beta \leq \alpha$ and $\alpha \leq \beta$. That is $\alpha=\beta$, then $\alpha \in \operatorname{Min}(X)$.

$\operatorname{Min}(Z)=\emptyset$ : Assume there exists an element $z \in \operatorname{Min}(Z)$. Since $\operatorname{Min}(Z) \subseteq Z$, $z \in Z=X \backslash Y$, that is $z \notin Y$. Note that $z \notin \operatorname{Min}(X)$, so there exists $x_{1} \in X$ with $x_{1} \neq z$ such that $x_{1} \leq z$.

We claim that $x_{1} \in Z$ i.e $x_{1} \notin Y$. Otherwise, if $x_{1} \in Y$, there exists $x_{2} \in \operatorname{Min}(X)$ such that $x_{2} \leq x_{1} \leq z$ which implies $z \in Y$, a contradiction.

(ii) Consider the definition of $Y$. For each $y \in Y$ there exists a minimal element $x \in$ $\operatorname{Min}(X)$ with $x \leq y$. By $(2), \operatorname{Min}(Y)=\operatorname{Min}(X)$. So $x \in \operatorname{Min}(Y)$. By Lemma 1.1 (i), $\operatorname{Min}(Y)$ is a maximal antichain of $Y$.

(iii) Assume $\operatorname{Min}(Y)$ is a maximal antichain and on the contrary, that there is an element $z \in Z$. So, $\operatorname{Min}(Y) \cup\{z\}$ is also an antichain by definition of $Y$. This is the desired contradiction.

Assume $Z=\emptyset$. Then by (i), $X=Y$. Using the definition of $Y$, there exists $x \in \operatorname{Min}(X)$ such that $x \leq y$. Thus $\operatorname{Min}(X) \neq \emptyset$. Since $X=Y$, there exists $y \in Y$ such that $y \leq x$. By Lemma 1.1 (i) $\operatorname{Min}(Y)$ is a maximal antichain of $Y=X$. 


\section{Singular ideal of incidence rings}

In this section, we will investigate the left singular ideal of an incidence ring. Moreover, similar arguments that appear here can be used to achieve analogous results for the right singular ideal.

We start by quoting some results that appeared in the literature. The following lemma is from [2], we give the proof for completeness of the argument in the sequel.

Lemma 2.1. ([2, Lemma 11]) Assume $r$ is a non-zero element of $R$, and $x, y \in X$ with $x \leq y$. Then ann $_{l}\left(r e_{x y}\right)$ is a left essential ideal of $I(X, R)$ if and only if ann $(r)$ is a left essential ideal of $R$.

Proof. First, note that $a n n_{l}\left(r e_{x y}\right)=\left\{g \in I(X, R): g(u, x) \in a n n_{l}(r)\right.$ for all $\left.u \in X\right\}$.

Assume $\operatorname{ann}_{l}(r)$ is a left essential ideal of $R$. Take any non-zero $f \in I(X, R)$.

Case 1: If $f(u, x)=0$ for all $u \in X$, then $f \in \operatorname{ann}_{l}\left(r e_{x y}\right)$. Thus $a_{n n}\left(r e_{x y}\right) \cap I(X, R) f \neq$ $\{0\}$.

Case 2: Otherwise, there exists $z \in X$ such that $f(z, x) \neq 0$. Let $S$ be the set of all $z \in X$ such that $f(z, x) \neq 0$. Then $\operatorname{ann}_{l}(r) \cap R f(z, x) \neq\{0\}$. So there exists $r_{z} \in R$ such that $r_{z} f(z, x) \in \operatorname{ann}_{l}(r), r_{z} f(z, x) \neq 0$. Now, construct $g \in I(X, R)$ where

$$
g(u, v)=\left\{\begin{array}{ccc}
r_{z} & \text { if } & u \leq z \text { and } v=z \\
0 & \text { otherwise }
\end{array} .\right.
$$

For any $u \in X, S \cap[u, x]$ is a finite set, as $X$ is locally finite, so

$$
g f(u, x)=\sum_{t} g(u, t) f(t, x)=\sum_{z \in S \cap[u, x]} g(u, z) f(z, x)=\sum_{z \in S \cap[u, x]} r_{z} f(z, x) \in \operatorname{ann}_{l}(r) .
$$

So $g f \in \operatorname{ann}_{l}\left(r e_{x y}\right)$. Now, we show $g f \neq 0$. There is a $z \in S$, and an interval $[z, x]$ such that $S \cap[z, x]=\{z\}$. Then $g f(z, x)=g(z, z) f(z, x)=r_{z} f(z, x) \neq 0$.

Then $g f \in a_{n n}\left(r e_{x y}\right) \cap I(X, R) f$. Hence $a n n_{l}\left(r e_{x y}\right)$ is a left essential ideal of $I(X, R)$.

Conversely, assume $a n n_{l}\left(r e_{x y}\right)$ is a left essential ideal of $I(X, R)$. Take any non-zero $a \in R$. Then $I(X, R)\left(a e_{x x}\right) \cap a n n_{l}\left(r e_{x y}\right) \neq 0$, so there exists $f \in I(X, R)$ such that $f a e_{x x} \neq 0$ and also $f a e_{x x} \in a n n_{l}\left(r e_{x y}\right)$. Hence there exists $u \in X$ such that $f(u, x) a \neq 0$ and also $f(u, x) a \in \operatorname{ann}_{l}(r)$. Therefore, $R a \cap a n_{l}(r) \neq 0$ and $a n_{l}(r)$ is left essential.

Now, we are ready to prove that for any $X$ and $R$, the finite support of $I\left(X, \operatorname{Sing}_{l}(R)\right)$ is contained in the singular ideal of $I(X, R)$.

Proposition 2.2. For any partially ordered set $X$ and ring $R$,

$$
F S\left(I\left(X, \operatorname{Sing}_{l}(R)\right)\right) \subseteq \operatorname{Sing}_{l}(I(X, R)) .
$$

Proof. Let $f$ be in the finite support of $\left.I\left(X, \operatorname{Sing}_{l}(R)\right)\right)$, then $f=\sum r_{x y} e_{x y}$ where $r_{x y} \in$ $\operatorname{Sing}_{l}(R)$ and the sum is over finitely many entries $(x, y)$ in $X \times X, x \leq y$. For any $(x, y)$ with $x \leq y$, ann $l\left(r_{x y}\right)$ is a left essential ideal of $R$. By Lemma $2.1 a n n_{l}\left(r_{x y} e_{x y}\right)$ is left essential ideal of $I(X, R)$, so $r_{x y} e_{x y}$ is in the left singular ideal of $I(X, R)$. Hence, $f \in \operatorname{Sing}_{l}(I(X, R))$.

For any $X$ and $R, \operatorname{Sing}_{l}(I(X, R)) \subset I\left(X, \operatorname{Sing}_{l}(R)\right)$, which is already proved in $[2$, Theorem 12, Proposition 13]. We include the proof here for the completeness of the argument.

Proposition 2.3. Assume $X$ is a locally finite partially ordered set, $R$ is any ring. Then

$$
\operatorname{Sing}_{l}(I(X, R)) \subset I\left(X, \operatorname{Sing}_{l}(R)\right) .
$$


Proof. Take $f \in \operatorname{Sing}_{l}(I(X, R))$ and $f \neq 0$, then $\operatorname{ann}_{l}(f)$ is left essential.

$$
\operatorname{ann}_{l}(f)=\bigcap_{x \in X} a n n_{l}\left(f e_{x x}\right) \subseteq \operatorname{ann}_{l}\left(f e_{x x}\right) \text { for all } x \in X .
$$

Hence, $a_{n n}\left(f e_{x x}\right)$ is a left essential ideal for all $x \in X$.

Since $f \neq 0$, there exists $f(x, y) \neq 0$ for some $(x, y) \in X \times X$. Let $r \in R, r \neq 0$ be arbitrary. Construct $r e_{x x} \in I(X, R)$. Then

$$
\operatorname{ann}_{l}\left(f e_{y y}\right) \cap I(X, R)\left(r e_{x x}\right) \neq\{0\} .
$$

So there exists $h \in I(X, R)$ such that $h\left(r e_{x x}\right) \neq 0$ and $h r e_{x x} \in a n n_{l}\left(f e_{y y}\right)$. That is, there exists $t \leq x$ such that

$$
\left(h r e_{x x}\right)(t, x)=h(t, x) r \neq 0
$$

and

$$
\sum_{v \leq z \leq y}\left(h r e_{x x}\right)(v, z) f(z, y)=0 \text { for all } v \leq x
$$

For $v=t$,

$$
0=\sum_{t \leq z \leq y}\left(h r e_{x x}\right)(t, z) f(z, y)=h(t, x) r f(x, y) .
$$

This implies $h(t, x) r \in \operatorname{ann}_{l}(f(x, y))$. So $h(t, x) r \in R r \cap \operatorname{ann}_{l}(f(x, y)) \neq\{0\}$. Then $\operatorname{ann}_{l}(f(x, y))$ is left essential in $R$. So $f(x, y) \in \operatorname{Sing}_{l}(R)$. Hence $f \in I\left(X, \operatorname{Sing}_{l}(R)\right)$.

Propositions 2.2 and 2.3 give the main result on the singular ideal of an incidence ring.

Theorem 2.4. For any locally finite partially ordered set $X$ and any ring $R$,

$$
F S\left(I\left(X, \operatorname{Sing}_{l}(R)\right)\right) \subseteq \operatorname{Sing}_{l}(I(X, R)) \subseteq I\left(X, \operatorname{Sing}_{l}(R)\right) .
$$

We turn our attention to sharpening the inclusion in Theorem 2.4 under some restrictions of $X$ or $R$ in the rest of this section. We analyze the conditions needed for $\operatorname{Sing}_{l}(I(X, R))=I\left(X, \operatorname{Sing}_{l}(R)\right)$.

In [2, Theorem 12], it is shown that $I(X, R)$ is (left) nonsingular if and only if $R$ is left non-singular. Although this result is a very simple observation, as we have quoted the statement throughout the text, we will state it as a proposition.

Proposition 2.5. ([2, Theorem 12]) $\operatorname{Sing}_{l}(I(X, R))=\{0\}$ if and only if $\operatorname{Sing}_{l}(R)=\{0\}$. Hence, in this case,

$$
\operatorname{Sing}_{l}(I(X, R))=I\left(X, \operatorname{Sing}_{l}(R)\right)=\{0\} .
$$

Now, we consider the left singular ideal of the incidence ring over a partially ordered set $X$ with $\operatorname{Min}(X)$ a maximal antichain and $\kappa(x)$ finite. We will need the dual version of the following lemma from [1] that we restate here.

Lemma 2.6. ([1, Lemma 1, Lemma 2]) Assume $A$ is a left ideal of $I(X, R)$. For any $x, y \in X$ with $x \leq y$,

(i) $A(x, y)=\{f(x, y) \in R: f \in A\}$ is a left ideal of $R$.

(ii) For $x \leq y \leq z$ in $X, A(x, y) \subset A(x, z)$.

(iii) If $A$ is an essential left ideal and $x \in \operatorname{Min}(X)$, then $C(x, y)=\left\{f(x, y) \in R: f \in R_{x y} \cap A\right\}$ is an essential left ideal of $R$ and $C(x, y) \subset$ $A(x, y)$.

(iv) If $A$ is an essential left ideal then there exists a family $\mathcal{E}$ of essential left ideals of $R$ such that for any $x \in \operatorname{Min}(X)$, and $y \in X$ with $x \leq y$, there exists $E_{x y} \in \mathcal{E}$ with $E_{x y} e_{x y} \subset A$. 
(v) Assume $\operatorname{Min}(X)$ is a maximal antichain and $\kappa(x)$ is finite for all $x \in X$. If there exists a family $\mathcal{E}$ of essential left ideals of $R$ such that for any $x \in \operatorname{Min}(X)$, and $y \in X$ with $x \leq y$, there exists $E_{x y} \in \mathcal{E}$ with $E_{x y} e_{x y} \subset A$, then $A$ is an essential left ideal.

This result is a special case of Theorem 2.4, where the equality is achieved:

Proposition 2.7. Assume $X$ is a locally finite partially ordered set where $\operatorname{Min}(X)$ is a maximal antichain and $\kappa(x)$ is finite for any $x \in \operatorname{Min}(X)$. Then, the left singular ideal of $I(X, R)$ is the incidence subring of $X$ over the left singular ideal of $R$. That is,

$$
\operatorname{Sing}_{l}(I(X, R))=I\left(X, \operatorname{Sing}_{l}(R)\right) \text {. }
$$

Proof. By Proposition 2.3, one containment is satisfied.

For the converse, take a non-zero element $f \in I\left(X, \operatorname{Sing}_{l}(R)\right)$. We want to show that $a_{n n}(f)$ is a left essential ideal in $I(X, R)$. By using Lemma 2.6, it is enough to show that there exists a collection $\mathcal{E}=\left\{A_{x y}: x \in \operatorname{Min}(X), x \leq y\right\}$ of left essential ideals in $R$, such that $A_{x y} e_{x y} \subset \operatorname{ann}_{l}(f)$ for all $x \in \operatorname{Min}(X), x \leq y$. Pick any $x, y$ with $x \in \operatorname{Min}(X)$ and $x \leq y$. Define

$$
A_{x y}=\bigcap_{v \in U(y)} \operatorname{ann}_{l}(f(y, v)) .
$$

Since $f(y, v) \in \operatorname{Sing}_{l}(R)$, $\operatorname{ann}_{l}(f(y, v))$ is left essential ideal of $R$ for all $v \in U(y)$. As, $|U(y)|=\kappa(y)$ is a finite set by Lemma 1.1 (ii), $A_{x y}$ is the finite intersection of left essential ideals. Hence, $A_{x y}$ is left essential. Moreover, take any $r \in A_{x y}=\bigcap a n n_{l}(f(y, v))$, to complete the proof we show that $r e_{x y} \in A_{x y} e_{x y}$ is in the left annihilator of $f$. Take any $(u, v)$,

$$
\left(r e_{x y} f\right)(u, v)=\left\{\begin{array}{ll}
r f(y, v) & \text { if } u=x, y \leq v \\
0 & \text { otherwise }
\end{array}=\left\{\begin{array}{ll}
0 & \text { if } u=x, y \leq v \\
0 & \text { otherwise }
\end{array}=0 .\right.\right.
$$

Hence $A_{x y} e_{x y} f=0$ and $A_{x y} e_{x y} \subset a n n_{l}(f)$.

When $X$ is finite, $\operatorname{Min}(X)$ is a maximal antichain by Remark 1.3. Clearly, any finite $X$ is upper finite. Hence, we get the following corollary which is already stated in [2, Proposition $13]$.

Corollary 2.8. Assume $X$ is finite, then

$$
\operatorname{Sing}_{l}(I(X, R))=I\left(X, \operatorname{Sing}_{l}(R)\right) \text {. }
$$

Another proof of Corollary 2.8 is that the finite support of $I\left(X, \operatorname{Sing}_{l}(R)\right)$ is $I\left(X, \operatorname{Sing}_{l}(R)\right)$ itself, when $X$ is finite. By Proposition 2.2, the result follows.

\section{Socle of incidence rings}

In this section, we will investigate the socle of an incidence ring. In order to calculate the left socle, we will look at the essential ideals of an incidence ring, which are already studied in [5]. Also, any dense ideal is essential, so the description of various dense ideals of an incidence algebra defined in the paper [3] will be useful.

The following constructions are from [3].

For any $x \in \operatorname{Min}(X)$, fix a left ideal $I_{x}$ of $R$ and define a subset $S\left(x, I_{x}\right)$ of $I(X, R)$ as

$$
S\left(x, I_{x}\right)=\left\{f \in I\left(X, I_{x}\right): f(u, v)=0 \text { if } u \neq x\right\} .
$$

Then the set

$$
C(I(X, R)):=\bigoplus_{x \in \operatorname{Min}(X)} S\left(x, I_{x}\right)=\bigoplus_{x \in \operatorname{Min}(X)}\left\{f \in I\left(X, I_{x}\right): f(u, v)=0 \text { if } u \neq x\right\}
$$


is a two-sided ideal of $I(X, R)$.

Lemma 3.1. Assume $X$ is a locally finite partially ordered set where $\operatorname{Min}(X)$ is a maximal antichain and $\left\{I_{x}: x \in \operatorname{Min}(X)\right\}$ is a collection of left essential ideals of $R$.

(i) $C(I(X, R))$ is a left essential ideal of $I(X, R)$.

(ii) Let $C$ be the intersection of all left essential ideals of the form $C(I(X, R))$. Then

$$
C=\bigoplus_{x \in \operatorname{Min}(X)} S\left(x, \operatorname{Soc}_{l}(R)\right)
$$

(iii) $\operatorname{Soc}_{l}(I(X, R)) \subset C$.

Proof. (i) Assume $\operatorname{Min}(X)$ is a maximal antichain. Then left ideals of the form $C(I(X, R))$ are left essential.

(ii) If $f \in C$, then $f=f_{x_{1}}+f_{x_{2}}+\cdots f_{x_{n}}$ where $f_{x_{i}} \in S\left(x_{i}, D_{i}\right)$ for any left essential ideal $D_{i}$ where $x_{i} \in \operatorname{Min}(X), i=1, \cdots, n$. So, $f_{x_{i}} \in S\left(x_{i}, S_{x_{l}}(R)\right)$ and $f \in$ $\bigoplus_{x \in \operatorname{Min}(X)} S\left(x, \operatorname{Soc}_{l}(R)\right)$. Similarly, $f \in \bigoplus_{x \in \operatorname{Min}(X)} S\left(x, \operatorname{Soc}_{l}(R)\right)$ implies $f$ is in any left essential ideal of the form $C(I(X, R))$, so $f \in C$.

(iii) Since $\operatorname{Soc}_{l}(I(X, R))$ is the intersection of all left essential ideals, the result follows.

When we consider the assumption that $\operatorname{Min}(X)$ is a maximal antichain and $\kappa(x)$ is finite for any $x \in \operatorname{Min}(X)$, we achieve the equality, i.e.,

$$
\operatorname{Soc}_{l}(I(X, R))=\bigoplus_{x \in \operatorname{Min}(X)} S\left(x, \operatorname{Soc}_{l}(R)\right) .
$$

Theorem 3.2. Assume $X$ is a locally finite partially ordered set where $\operatorname{Min}(X)$ is a maximal antichain and $\kappa(x)$ is finite for any $x \in \operatorname{Min}(X)$. Then,

$$
\operatorname{Soc}_{l}(I(X, R))=\bigoplus_{x \in \operatorname{Min}(X)} S\left(x, \operatorname{Soc}_{l}(R)\right)
$$

Proof. Assume $\operatorname{Min}(X)$ is a maximal antichain, then Lemma 3.1 (iii) gives one inclusion. Also, by hypothesis $\kappa(x)$ is finite for any $x \in \operatorname{Min}(X)$. Now, let $f \in \bigoplus_{x \in \operatorname{Min}(X)} S\left(x, \operatorname{Soc}_{l}(R)\right)$, and $K$ be any left essential ideal of $I(X, R)$.

Let $M$ be a subset of $\operatorname{Min}(X)$, say $M=\left\{x_{1}, x_{2}, \ldots, x_{n}\right\}$. Now, $f=\sum_{x \in M} f_{x}$ where $f_{x} \in S\left(x_{i}, \operatorname{Soc}_{l}(R)\right)$ for some $x_{i} \in M, i=1, \cdots, n$. Let $x \in M$ then

$$
f_{x}=\sum_{x \leq y} \alpha_{y} e_{x y} \text { where } \alpha_{y} \in \operatorname{Soc}_{l}(R) .
$$

Since $\kappa(x)<\infty$, this sum is finite. Then

$$
C(x, y)=\left\{r \in R: r e_{x y} \in R e_{x y} \cap K\right\}
$$

is a left essential ideal of $R$ by Lemma 2.6 (iii). So $\operatorname{Soc}_{l}(R) \subseteq C(x, y)$. Since $\alpha_{y} \in$ $\operatorname{Soc}_{l}(R) \subseteq C(x, y)$

$$
\alpha_{y} e_{x y} \in C(x, y) e_{x y}=R e_{x y} \cap K \subseteq K
$$

and $f_{x} \in K$, hence $f \in K$. For any left essential ideal $K$,

$$
\bigoplus_{x \in \operatorname{Min}(X)} S\left(x, \operatorname{Soc}_{l}(R)\right) \subseteq K
$$

and

$$
\bigoplus_{x \in \operatorname{Min}(X)} S\left(x, \operatorname{Soc}_{l}(R)\right) \subseteq \bigcap_{\text {all essential } K} K=\operatorname{Soc}_{l}(I(X, R)) .
$$

This completes the proof. 
We provide an example for the previous theorem.

Example 3.3. The partially ordered set $\cup C_{n}$ is defined to be the set

$$
X=\left\{x_{11}, x_{21}, x_{22}, x_{31}, x_{32}, x_{33}, x_{41}, \cdots\right\}
$$

with the relation that $x_{i j} \leq x_{k l}$ whenever $i=k$ and $j \leq l$. The set $\cup C_{n}$ is an example of an unbounded partially ordered set with no infinite chain.

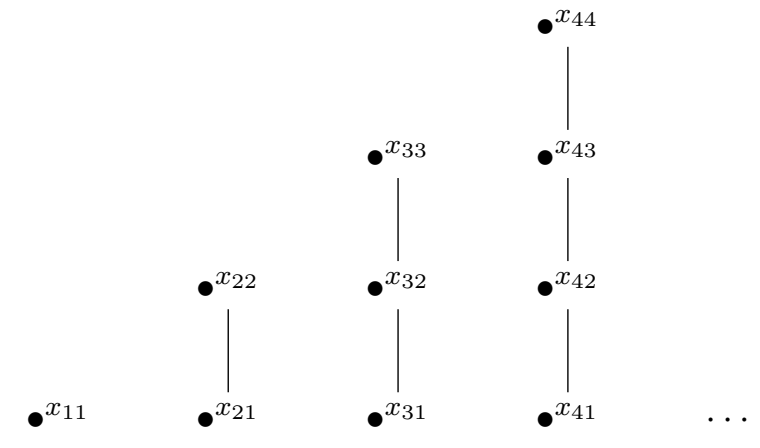

By Theorem 3.2,

$$
\operatorname{Soc}_{l}(I(X, R))=\bigoplus_{x \in \operatorname{Min}(X)} S\left(x, \operatorname{Soc}_{l}(R)\right) .
$$

In [3, Lemma 4], for each $n \in \mathbb{N}$, a collection of left dense ideals $Z_{n}$ is constructed as follows:

$$
Z_{n}:=\{f \in I(X, R): f(x, y)=0 \text { if }|[x, y]| \leq n, \text { and } x \notin \operatorname{Min}(X)\} .
$$

The following lemma is proved in [3, Lemma 4].

Lemma 3.4. $Z_{n}$ is a two-sided ideal of $I(X, R)$.

(i) $Z_{n}$ is a left essential ideal of $I(X, R)$.

(ii) Let $Z$ be the intersection of all $Z_{n}$. Then $\operatorname{Soc}_{l}(I(X, R)) \subset Z$ where

$$
\begin{gathered}
Z=\bigcap_{n \in \mathbb{N}} Z_{n}=\{f \in I(X, R): f(x, y)=0 \text { for all } y \in X, x \notin \operatorname{Min}(X)\} \\
=\prod_{x \in \operatorname{Min}(X)} S(x, R) .
\end{gathered}
$$

(iii) If $\operatorname{Min}(X)$ is a finite set, then $Z=\bigoplus_{x \in \operatorname{Min}(X)} S(x, R)$.

(iv) If $\operatorname{Min}(X)=\emptyset$, then $Z=\{0\}$.

Proof. It is shown in [3, Lemma 4] that $Z_{n}$ is a left dense ideal of $I(X, R)$. Any dense ideal is essential. The rest is trivial.

Proposition 3.5. Let $R$ be any ring, $X$ be a locally finite partially ordered set where $\operatorname{Min}(X)=\emptyset$. Then,

$$
\operatorname{Soc}_{l}(I(X, R))=\{0\} .
$$

Proof. By Lemma 3.4 (ii) and (iv), the result follows.

In the following example, $X$ is upper finite, but there are no minimal elements.

Example 3.6. Let $X=\mathbb{Z}^{-}, R$ be any ring. Then $\operatorname{Min}(X)=\emptyset$. By Proposition 3.5, $\operatorname{Soc}_{l}(I(X, R))=\{0\}$.

Our next theorem will restrict $R$ to calculate the socle of the incidence ring. Hence, we define a collection of sets $D_{n}$ as follows:

$$
D_{n}:=\left\{f \in I\left(X, \operatorname{Soc}_{l}(R)\right): f(x, y)=0 \text { if }|[x, y]| \leq n \text {, and } x \notin \operatorname{Min}(X)\right\} .
$$


Lemma 3.7. (i) For each $n \in \mathbb{N}, D_{n}$ is a two-sided ideal of $I(X, R)$.

(ii) If $R$ is Artinian and nonsingular, then for each $n \in \mathbb{N}, D_{n}$ is a left essential ideal of $I(X, R)$.

(iii) If $R$ is Artinian and nonsingular, then for $D=\bigcap_{n \in \mathbb{N}} D_{n}$ we have $\operatorname{Soc}_{l}(I(X, R)) \subset$ D. More precisely,

$$
\begin{gathered}
D=\left\{f \in I\left(X, \operatorname{Soc}_{l}(R)\right): f(x, y)=0 \text { for all } y \in X, x \notin \operatorname{Min}(X)\right\} \\
=\prod_{x \in \operatorname{Min}(X)} S\left(x, \operatorname{Soc}_{l}(R)\right) .
\end{gathered}
$$

(iv) If $R$ is Artinian and nonsingular and $\operatorname{Min}(X)$ is a finite set, then

$$
D=\bigoplus_{x \in \operatorname{Min}(X)} S\left(x, S_{\text {oc }}(R)\right)
$$

Proof. (i) For any $g \in I(X, R)$ and $f \in D_{n},(g f)(x, y)=\sum_{x \leq z \leq y} g(x, z) f(z, y)$. For $x \notin$ $\operatorname{Min}(X)$ and $|[x, y]| \leq n$, we also have $f(x, y)=0$. For all $z \leq y$, since $z \notin \operatorname{Min}(X)$ and $|[z, y]| \leq n$ we get $f(z, y)=0$. Then $(g f)(x, y)=0$ and $g f \in D_{n}$.

(ii) We first show that $D_{n}$ is a left dense ideal. Let $(l, z) \in X \times X$ with $l \leq z$.

Case 1: Assume that there exists $x \in \operatorname{Min}(X)$ such that $x \leq l \leq z$. For all $\alpha \in$ $\operatorname{Soc}_{l}(R), \alpha e_{x l} \in D_{n}$ and for any $g \in A n n_{r}\left(D_{n}\right)$,

$$
\left(\alpha e_{x l} g\right)(x, z)=0
$$

then

$$
0=\left(\alpha e_{x l} g\right)(x, z)=\sum_{x \leq y \leq z} \alpha e_{x l}(x, y) g(y, z)=\alpha g(l, z)
$$

Hence

$$
g(l, z) \in \operatorname{Ann}_{r}\left(\operatorname{Soc}_{l}(R)\right) .
$$

Since $R$ is nonsingular, $\left.\operatorname{Soc}_{l}(R)\right)=R$ and $\operatorname{Ann}_{r}\left(\operatorname{Soc}_{l}(R)\right)=0$. Hence,

$$
g(l, z)=0 .
$$

Case 2: Now assume that there in no $x \in \operatorname{Min}(X)$ with $x \leq l$. For the case $|[l, z]|>n$, take $x_{1} \in X$ such that $\left|\left[x_{1}, l\right]\right|>n$ with $g \in \operatorname{Ann}_{r}\left(D_{n}\right)$ and for all $\alpha \in \operatorname{Soc}_{l}(R)$,

$$
\left(\alpha e_{x_{1} l} g\right)(x, z)=0 \text {. }
$$

We again have

$$
0=\left(\alpha e_{x l} g\right)(x, z)=\sum_{x \leq y \leq z} \alpha e_{x l}(x, y) g(y, z)=\alpha g(l, z) .
$$

Then by the same manner

$$
g(l, z)=0 .
$$

Hence $\operatorname{Ann}_{r}\left(D_{n}\right)=0$ which means that $D_{n}$ is a dense ideal of $I(X, R) \dot{)}$. Since a dense ideal is essential, $D_{n}$ is essential. Further, $R$ is non-singular, hence all essential ideals are dense.

(iii) \& (iv) are obvious.

Theorem 3.8. Let $X$ be a locally finite partially ordered set. Assume that $R$ is an Artinian and nonsingular ring, $\operatorname{Min}(X)$ is finite and $\kappa(x)$ is finite for all $x \in X$, then

$$
\operatorname{Soc}_{l}(I(X, R))=\bigoplus_{x \in \operatorname{Min}(X)} S\left(x, \operatorname{Soc}_{l}(R)\right) .
$$


Proof. As $\operatorname{Min}(X)$ is a finite set, so by Lemma 3.7 (iii) and (iv),

$$
\operatorname{Soc}_{l}(I(X, R)) \subseteq \bigoplus_{x \in \operatorname{Min}(X)} S\left(x, \operatorname{Soc}_{l}(R)\right) .
$$

Also, by hypothesis $\kappa(x)$ is finite for any $x \in X$. Now, let $f \in \bigoplus_{x \in \operatorname{Min}(X)} S\left(x, \operatorname{Soc}_{l}(R)\right)$, and $K$ be any left essential ideal of $I(X, R)$. Let $M$ be a subset of $\operatorname{Min}(X)$, say $M=$ $\left\{x_{1}, x_{2}, \ldots, x_{n}\right\}$.

Now, $f=\sum_{x \in M} f_{x}$ where $f_{x} \in S\left(x_{i}, \operatorname{Soc}_{l}(R)\right)$ for some $x_{i} \in M, i=1, \cdots, n$. Let $x \in M$ then

$$
f_{x}=\sum_{x \leq y} \alpha_{y} e_{x y} \text { where } \alpha_{y} \in \operatorname{Soc}_{l}(R) .
$$

Since $\kappa(x)<\infty$, this sum is finite. Then

$$
C(x, y)=\left\{r \in R: r e_{x y} \in R e_{x y} \cap K\right\}
$$

is a left essential ideal of $R$ by Lemma 2.6 (iii). So $\operatorname{Soc}_{l}(R) \subseteq C(x, y)$. Since $\alpha_{y} \in$ $\operatorname{Soc}_{l}(R) \subseteq C(x, y)$,

$$
\alpha_{y} e_{x y} \in C(x, y) e_{x y}=R e_{x y} \cap K \subseteq K
$$

and $f_{x} \in K$, hence $f \in K$. For any left essential ideal $K$,

$$
\bigoplus_{x \in \operatorname{Min}(X)} S\left(x, \operatorname{Soc}_{l}(R)\right) \subseteq K,
$$

and

$$
\bigoplus_{x \in \operatorname{Min}(X)} S\left(x, \operatorname{Soc}_{l}(R)\right) \subseteq \bigcap_{\text {all essential } K} K=\operatorname{Soc}_{l}(I(X, R)) .
$$

This completes the proof.

Example 3.9. Let $R$ be an Artinian nonsingular ring and $X=Y \sqcup Z$ where $Y=$ $\left\{z_{1}, y_{1}, y_{2}, y_{3}, y_{4}\right\}$ and $Z=\left\{z_{i}: i \in \mathbb{N}, i \geq 2\right\}$ be a partially ordered set with the relations $y_{i} \leq z_{1}$, for $1 \leq i \leq 4$ and $z_{i+1} \leq z_{i}$ for all $i \in \mathbb{N}$. The Hasse diagram of $X$ is

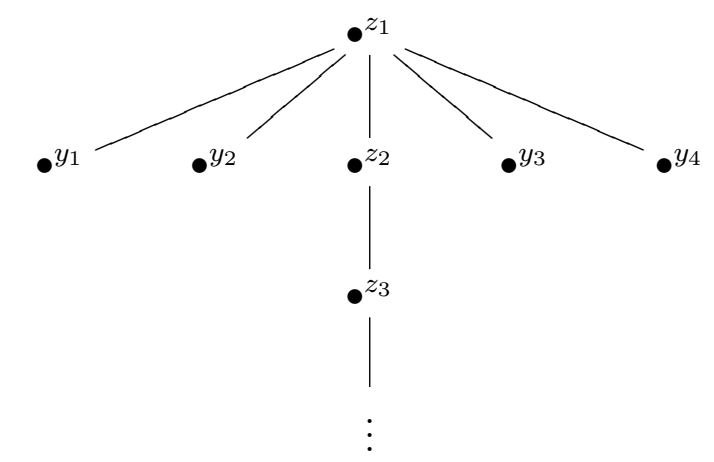

Now, $\operatorname{Min}(X)=\left\{y_{1}, y_{2}, y_{3}, y_{4}\right\}$ and by Theorem 3.8,

$$
\operatorname{Soc}_{l}(I(X, R))=\bigoplus_{x \in \operatorname{Min}(X)} S\left(x, \operatorname{Soc}_{l}(R)\right)=\bigoplus_{i=1}^{4} S\left(y_{i}, \operatorname{Soc}_{l}(R)\right) .
$$

Now we will use the minimal left ideals of the ring to calculate the socle. Recall the partially ordered sets we defined in Section 1 which we use in the proofs of the following results. The proof of Lemma 3.10 is given by Eugene Spiegel in a private conversation.

Let $X$ be a locally finite partially ordered set with a subset $Y$ defined as:

$$
Y=\{y \in X: \text { there is a } x \in \operatorname{Min}(X) \text { with } x \leq y\} .
$$

Then, $X=Y \sqcup(X \backslash Y)$ and let $Z=X \backslash Y$. 
Lemma 3.10. Let $X$ be a locally finite partially ordered set. If $N$ is a minimal left ideal of $I(X, R)$, then $N$ is of the form $R f$ for some $f \in S(x, R)$ and $x \in \operatorname{Min}(X)$.

Proof. Assume $N$ is a minimal left ideal of $I(X, R)$. We first prove that for any $f \in N$, $f(u, v)=0$ for all $u \in Z$. Assume on the contrary that there exists an $f \in N$ such that $f(n, m) \neq 0$ for some $n \in Z$ and $m \in X$. Since $n \in Z$, there does not exist $x \in \operatorname{Min}(X)$ such that $x \leq n$. So there exists $t_{1} \in X$ such that $t_{1}<n$. Then $t_{1} \in Z$ and $Z$ does not have any minimal elements. So $t_{1}$ does not have any minimal element smaller than in the order, otherwise $n$ would have had the same minimal elements, contradicting the fact that $n \in Z$. In a similar manner, there exists $t_{2} \in Z$ such that $t_{2}<t_{1}<n$. Hence, we conclude that there exists a non-terminating sequence $\left\{t_{i}\right\}_{i \in \mathbb{N}}$ with $\ldots<t_{i+1}<t_{i}<\ldots<t_{2}<t_{1}<n$. There are infinitely many $t \in Z$ such that $t \leq n$.

Take $e_{t n} \in I(X, R)$ and let $g_{t}=e_{t n} f$ then

$$
g_{t}(x, y)=e_{t n} f(x, y)=\sum_{x \leq z \leq y} e_{t n}(x, z) f(z, y)=\left\{\begin{array}{cl}
0 & \text { if } \quad x \neq t \text { or } n \not \leq y \\
f(n, y) & \text { if } x=t \text { and } n \leq y
\end{array} .\right.
$$

Notice that for any $k \in X$ such that $t<k \leq m, g_{t}(k, m)=e_{t n} f(k, m)=0$ since $k \neq t$. For $k=t, g_{t}(t, m)=e_{t n}(t, n) f(n, m)=f(n, m) \neq 0$.

Take any $h \in\left\langle g_{t}\right\rangle_{l}$, then $h=h^{\prime} g_{t}$ for some $h^{\prime} \in I(X, R)$. If $t<k \leq m$, then

$$
h(k, m)=\sum_{k \leq z \leq m} h^{\prime}(k, z) g_{t}(z, m)=0
$$

since $z \neq t$. So for $h \in\left\langle g_{t}\right\rangle_{l}, h(k, m)=0$ for all $k$ with $t<k \leq m$. Hence, if $h \in \bigcap_{t<n}\left\langle g_{t}\right\rangle_{l}$, then $h(u, m)=0$ for all $u \leq m$.

On the other hand, $g_{t} \in\langle f\rangle_{l}=N$. Since $N$ is a minimal left ideal, all nonzero elements of $N$ are generators. For all $t<n,\left\langle g_{t}\right\rangle_{l}=\langle f\rangle_{l}=N$ and $f \in\left\langle g_{t}\right\rangle_{l}$ for all $t$. Hence, $f \in \bigcap_{t<n}\left\langle g_{t}\right\rangle_{l}$ and $f(n, m) \neq 0$, this is the desired contradiction.

Moreover, $f \in I(Y, R)$ for all $f \in N$. If $u \in Z$ then $f(u, v)=0$ for all $f \in N$. So $N \subseteq I(Y, R)$. Then for some $u \in Y$, we get $f(u, v) \neq 0$, that is there is $x \in \operatorname{Min}(X)$ with $x \leq u$. Take $e_{x} \in I(X, R)$ then $e_{x} f \in I(X, R)$ and

$$
e_{x} f(u, v)=\left\{\begin{array}{ccc}
e_{x}(x, x) f(x, v) & \text { if } \quad x=u \\
0 & \text { if } \quad x \neq u
\end{array} .\right.
$$

Then $e_{x} f \subseteq S(x, R)$ and $\left\langle e_{x} f\right\rangle_{l} \in S(x, R)$. As $N$ is a minimal left ideal of $I(X, R)$, we get $\left\langle e_{x} f\right\rangle_{l}=N=\langle f\rangle_{l}$. Thus $f \in\left\langle e_{x} f\right\rangle_{l} \subseteq S(x, R)$ and $N=\langle f\rangle_{l}=R f$.

Lemma 3.11. Assume $A$ is a minimal left ideal of $R, x \in \operatorname{Min}(X)$ and $f \in S(x, R)$. Then any non-zero $A f$ is a minimal left ideal of $I(X, R)$.

Proof. Let $A$ be a minimal left ideal of $R$ and $f \in S(x, R)$ for some $x \in \operatorname{Min}(X)$. Consider $A f=\{a f: a \in A\}$ with $A f \neq 0$. Then for any $g \in I(X, R)$,

$$
g(a f)(u, v)=\left\{\begin{array}{ccc}
g(x, x) a f(x, v) & \text { if } u=x \\
0 & \text { if } u \neq x
\end{array} .\right.
$$

Hence $g(a f)=(g(x, x) a) f \in A f$ as $A$ is a left ideal of $R$. Af is clearly a left ideal of $I(X, R)$.

Take $a, b \in A$ such that $a f$ and $b f$ are non-zero elements of $A f$. Now, $A$ is a minimal left ideal so $\langle a f\rangle=\langle b f\rangle=A$. Then there exist $r, s \in R$ such that $a=r b$ and $b=s a$. So $a f=(r b) f=\left(r e_{x}\right)(b f) \in\langle b f\rangle_{l}$, which implies $\langle a f\rangle_{l} \subseteq\langle b f\rangle_{l}$. Similarly, $b f=(s a) f=$ $\left(s e_{x}\right)(a f) \in\langle a f\rangle_{l}$, which implies $\langle b f\rangle_{l} \subseteq\langle a f\rangle_{l}$. Hence $\langle b f\rangle_{l}=\langle a f\rangle_{l}$. Any nonzero element of $A f$ is a generator, that is $A f$ is a minimal left ideal of $I(X, R)$. 
Lemma 3.12. Assume $N$ is a minimal left ideal of $I(X, R)$, then $N$ is a minimal left ideal of $I(Y, R)$.

Proof. Asuume that $N$ is a minimal left ideal of $I(X, R)$, then $N=R f$ where $f \in S(x, R)$, for some $x \in \operatorname{Min}(X)$ by Lemma 3.10. Since for all $g \in I(Y, R)$ we have $g N \subseteq N$, clearly $N$ is a left ideal of $I(Y, R)$.

Pick any non-zero $a f \in N$ for some $a \in R$. Then the left ideal generated in $I(Y, R)$ is $\langle a f\rangle_{I(Y, R)} \subseteq N$. Since $f \in S(x, R)$, for all $h \in I(Y, R)$ we get

$$
\langle a f\rangle_{I(Y, R)}=\{h a f: h \in I(Y, R)\}=\{(h(x, x) a) f: h(x, x) \in R\}=R(a f)=(R a) f .
$$

Similarly,

$$
\langle a f\rangle_{I(X, R)}=\{h a f: h \in I(X, R)\}=\{(h(x, x) a) f: h(x, x) \in R\}=(R a) f .
$$

So, the left ideal generated by $\langle a f\rangle$ in $I(Y, R)$ is equal to the the left ideal generated by $\langle a f\rangle$ in $I(X, R)$. As $N$ is a minimal left ideal of $I(X, R), N=\langle a f\rangle_{I(X, R)}=\langle a f\rangle_{I(Y, R)}$. Hence, $N$ is a minimal left ideal in $I(Y, R)$.

Let $\mathfrak{M}$ be the collection of all minimal left ideals of $I(X, R)$ and $\mathfrak{N}$ be the collection of all minimal left ideals of $I(Y, R)$. By Lemma $3.12, \mathfrak{M} \subseteq \mathfrak{N}$ and

$$
\operatorname{Soc}_{l}(I(X, R))=\bigoplus_{N \in \mathfrak{M}} N \subseteq \bigoplus_{N \in \mathfrak{N}} N=\operatorname{Soc}_{l}(I(Y, R)) .
$$

Moreover, by Lemma 1.4, $\operatorname{Min}(Y)=\operatorname{Min}(X)$ is a maximal antichain in $Y$ and by Lemma 3.1

$$
\operatorname{Soc}_{l}(I(Y, R)) \subseteq \bigoplus_{x \in \operatorname{Min}(X)} S\left(x, \operatorname{Soc}_{l}(R)\right)
$$

Thus,

$$
\operatorname{Soc}_{l}(I(X, R)) \subseteq \bigoplus_{x \in \operatorname{Min}(X)} S\left(x, \operatorname{Soc}_{l}(R)\right) .
$$

Let $\mathfrak{A}$ be the collection of all minimal left ideals of $R$. By Lemma 3.11, for any minimal left ideal $A$ of $R$ with $x \in \operatorname{Min}(X)$ and $f \in S(x, R)$, any non-zero $A f$ is a minimal left ideal of $I(X, R)$. Hence,

$$
\bigoplus_{x \in \operatorname{Min}(X)} \bigoplus_{A \in \mathfrak{A}} \bigoplus_{f \in S(x, R)} A f \subseteq \operatorname{Soc}_{l}(I(X, R))
$$

Combining (3.1) and (3.2) gives the following result:

Theorem 3.13. Let $X$ be a locally finite partially ordered set and $R$ be a ring with unity. Then

$$
\bigoplus_{x \in \operatorname{Min}(X)} \bigoplus_{A \in \mathfrak{A}} \bigoplus_{f \in S(x, R)} A f \subseteq \operatorname{Soc}_{l}(I(X, R)) \subseteq \bigoplus_{x \in \operatorname{Min}(X)} S\left(x, \operatorname{Soc}_{l}(R)\right)
$$

where $\mathfrak{A}$ is the collection of all minimal left ideals of $R$.

We further assume that $R$ is a commutative ring.

Lemma 3.14. Let $R$ be a commutative ring and $A$ be a minimal ideal of $R$. Let $X$ be a locally finite partially ordered set with $x \in \operatorname{Min}(X)$. Then for any $g \in S(x, A)$, the left ideal generated by $g$ is a minimal left ideal of $I(X, R)$. Moreover

$$
\bigoplus_{g \in S(x, A)}\langle g\rangle_{l}=S(x, A) .
$$


Proof. Consider $g \in S(x, A)$, where $x \in \operatorname{Min}(X)$,f or any $u, v \in X$,

$$
g(u, v)=\left\{\begin{array}{l}
a_{v} \quad \text { if } \quad u=x, v \geq u \\
0 \quad \text { if } \quad u \neq x
\end{array}\right.
$$

where $a_{v} \in A$. We would like to show that the left ideal generated by $g$ is a minimal left ideal of $I(X, R)$. One can write the left ideal generated by $g$ as:

$$
\langle g\rangle_{l}=\{f \in I(X, R): f=a g \text { for some } a \in R\}
$$

Fix some $f \in\langle g\rangle_{l}$. That is, $f=a g$ for some fixed $a \in R$. Then for any $u, v \in X$, $f(u, v)=\left\{\begin{array}{l}a a_{v} \quad \text { if } \quad u=x, v \geq u \\ 0 \quad \text { if } \quad u \neq x\end{array} \quad\right.$ where $a_{v}=g(x, v) \in A$.

Now, for any $v, a_{v} \in A$ and $A$ is an (minimal) ideal of $R$, implies that $a a_{v} \in A$. Clearly, $\left\langle a a_{v}\right\rangle \subseteq A$. If $a a_{v}$ is non-zero, then by the minimality of $A$, we get $\left\langle a a_{v}\right\rangle=A$,

$$
\left\langle a a_{v}\right\rangle=A=\left\langle a_{v}\right\rangle=\left\langle a_{w}\right\rangle .
$$

For each non-zero $a_{v} \in A$, we can generate $A$ with $\left\langle a_{v}\right\rangle$. That is, there exists $r_{0} \in R$ such that $a_{v}=r_{0} a a_{v}$, and there exists $r_{w} \in R$ such that $a_{w}=r_{w} a_{v}$. For any $g \in S(x, A)$, $g(x, v)=a_{v}=r_{0} a a_{v}=r_{0} f(x, v)$. Since $R$ is commutative, $g(x, w)=a_{w}=r_{w} a_{v}=$ $r_{w}\left(r_{0} a\right) a_{v}=\left(r_{0} a\right) r_{w} a_{v}=\left(r_{0} a\right) a_{w}=r_{0}\left(a a_{w}\right)=r_{0} f(x, w)$. Hence

$$
\begin{array}{cl}
g(x, v)=r_{0} f(x, v) & \text { for all } v, \\
g(u, v)=0=r_{0} f(u, v) & \text { if } u \neq x .
\end{array}
$$

So $g=r_{0} e_{x x} f \in\langle f\rangle_{l}$. Thus

$$
\langle g\rangle_{l} \subseteq\langle f\rangle_{l} \subseteq\langle g\rangle_{l} .
$$

That is $\langle f\rangle_{l}=\langle g\rangle_{l}$. Hence we proved the left ideal generated by $g$ is a minimal left ideal of $I(X, R)$.

When $R$ is a commutative ring, by Lemma 3.14, we conclude that

$$
\bigoplus_{A \in \mathfrak{A}} \bigoplus_{x \in \operatorname{Min}(X)} S(x, A) \subseteq \operatorname{Soc}_{l}(I(X, R)) .
$$

Thus, Theorem 3.13 becomes

$$
\bigoplus_{A \in \mathfrak{A}} \bigoplus_{x \in \operatorname{Min}(X)} S(x, A) \subseteq \operatorname{Soc}_{l}(I(X, R)) \subseteq \bigoplus_{x \in \operatorname{Min}(X)} S(x, \operatorname{Soc}(R)) .
$$

Theorem 3.15. Assume $R$ is a commutative ring with finitely many minimal ideals and $X$ is a locally finite partially ordered set. Then

$$
\operatorname{Soc}_{l}(I(X, R))=\bigoplus_{x \in \operatorname{Min}(X)} S(x, \operatorname{Soc}(R)) .
$$

Proof. By Theorem 3.13, $\operatorname{Soc}_{l}(I(X, R)) \subseteq \bigoplus_{x \in M i n(X)} S(x, \operatorname{Soc}(R))$. For the converse inclusion, we use Lemma 3.14. Since $\operatorname{Soc}_{l}(I(X, R))$ is the sum of all minimal left ideals of $I(X, R)$,

$$
\bigoplus_{x \in \operatorname{Min}(X)} \bigoplus_{A} S(x, A) \subseteq \operatorname{Soc}_{l}(I(X, R))
$$

where $A$ ranges over all minimal ideals of $R$.

If $R$ has finitely many minimal ideals $\left\{A_{i}\right\}_{i=1}^{n}$, then $\bigoplus_{i=1}^{n} A_{i}=\operatorname{Soc}(R)$. Now, we will prove that for any $x \in \operatorname{Min}(X)$

$$
S(x, S o c(R))=\bigoplus_{i=1}^{n} S\left(x, A_{i}\right)
$$


and hence deduce

$$
\bigoplus_{x \in \operatorname{Min}(X)} S(x, \operatorname{Soc}(R)) \subseteq \operatorname{Soc}_{l}(I(X, R))
$$

Fix $x \in \operatorname{Min}(X)$. Given any $f \in S(x, \operatorname{Soc}(R))$ and $y \in X$, we have $f(x, y) \in \operatorname{Soc}(R)=$ $\bigoplus_{i=1}^{n} A_{i}$. That is

$$
f(x, y)=\sum_{i=1}^{n} a_{i}^{y}
$$

where $a_{i}^{y} \in A_{i}$. Define the functions $f_{i} \in I(X, R)$ for $i=1, \ldots, n$ as:

$$
f_{i}(u, y)=\left\{\begin{array}{ll}
0 & \text { if } u \neq x \\
a_{i}^{y} & \text { if } u=x,
\end{array} \text { for any } y \in X .\right.
$$

So $f_{i} \in S\left(x, A_{i}\right)$, hence $f=\sum_{i=1}^{n} f_{i} \in \bigoplus_{i=1}^{n} S\left(x, A_{i}\right)$. Clearly, $\bigoplus_{i=1}^{n} S\left(x, A_{i}\right)$ is contained in $S(x, S o c(R))$. This completes the proof.

We want to state that when $R$ is chosen to be a field or a commutative ring with finitely many minimal ideals, or $\mathbb{Z}$, socle and singular ideal are easy to compute.

Example 3.16. Let $X=\mathbb{Z}^{+}$, then $\operatorname{Min}(X)=\{1\}$ is a singleton and $\operatorname{Min}(X)$ is a maximal antichain, but $\kappa(x)$ is not finite for any $x$.

- if $R=\mathbb{Z}, \operatorname{Sing}(\mathbb{Z})=\{0\}=\operatorname{Soc}(\mathbb{Z})$.

By Proposition 2.5, $\operatorname{Sing}(I(X, \mathbb{Z}))=\{0\}$ and by Lemma 3.1,

$\operatorname{Soc}_{l}(I(X, \mathbb{Z})) \subset S(1, \operatorname{Soc}(\mathbb{Z}))=\{0\}$.

- $R=F$ is a field, then $\operatorname{Sing}(F)=\{0\}, \operatorname{Soc}(F)=F$.

By Proposition 2.5, Sing $(I(X, F))=\{0\}$ and since $F$ has a unique minimal ideal $F$ itself by Teorem 3.15 ,

$$
\operatorname{Soc}_{l}(I(X, F))=S(1, F) .
$$

- $R$ is a commutative ring with finitely many minimal ideals, Then $\operatorname{Soc}_{l}\left(I\left(\mathbb{Z}^{+}, R\right)\right)=$ $S(1, \operatorname{Soc}(R))$ by Theorem 3.15 .

We conclude this section by describing the right socle of $I(X, R)$ and give an example of an incidence ring having the same left and right socle.

For any $x \in \operatorname{Max}(X)$, fix a right ideal $I_{x}$ of $R$ and define $T\left(x, I_{x}\right)$ as

$$
T\left(x, I_{x}\right)=\left\{f \in I\left(X, I_{x}\right): f(u, v)=0 \text { if } v \neq x\right\} .
$$

Then, we form

$$
B(I(X, R)):=\bigoplus_{x \in \operatorname{Max}(X)} T\left(x, I_{x}\right)=\bigoplus_{x \in \operatorname{Max}(X)}\left\{f \in I\left(X, I_{x}\right): f(u, v)=0 \text { if } v \neq x\right\}
$$

which is a two-sided ideal of $I(X, R)$.

Equivalent to Lemma 3.1, we have the following results.

Lemma 3.17. Assume $X$ is a locally finite partially ordered set where $\operatorname{Max}(X)$ is a maximal antichain and $\left\{I_{x}: x \in \operatorname{Max}(X)\right\}$ is a collection of right essential ideals of $R$.

(1) $B(I(X, R))$ is a right essential ideal of $I(X, R)$.

(2) Let $B$ be the intersection of all right essential ideals of the form $B(I(X, R))$. Then

$$
B=\bigoplus_{x \in \operatorname{Max}(X)} T\left(x, \operatorname{Soc}_{r}(R)\right)
$$

(3) $\operatorname{Soc}_{r}(I(X, R)) \subset B$.

Under the assumption that $\operatorname{Max}(X)$ is a maximal antichain and $\lambda(x)$ is finite for any $x \in \operatorname{Max}(X)$, the description of the right socle follows. 
Theorem 3.18. Assume $X$ is a locally finite partially ordered set where $\operatorname{Max}(X)$ is a maximal antichain and $\lambda(x)$ is finite for any $x \in \operatorname{Max}(X)$. Then,

$$
\operatorname{Soc}_{r}(I(X, R))=\bigoplus_{x \in \operatorname{Max}(X)} T\left(x, \operatorname{Soc}_{r}(R)\right) .
$$

When $X$ is a finite partially ordered set, the hypotheses of Theorem 3.2 and Theorem 3.18 are satisfied. As an immediate corollary, the left and the right socle of the incidence ring are given explicitly as

$$
\begin{aligned}
\operatorname{Soc}_{l}(I(X, R)) & =\bigoplus_{x \in \operatorname{Min}(X)} S\left(x, \operatorname{Soc}_{l}(R)\right), \\
\operatorname{Soc}_{r}(I(X, R)) & =\bigoplus_{x \in \operatorname{Max}(X)} T\left(x, \operatorname{Soc}_{r}(R)\right) .
\end{aligned}
$$

This leads to constructing examples of incidence rings with distinct left and right socles.

Example 3.19. Let $X=\{x, y, z\}$ be a partially ordered set with the non-reflexive relations $x \leq y, x \leq z$. Hence, Hasse diagram of $X$ is

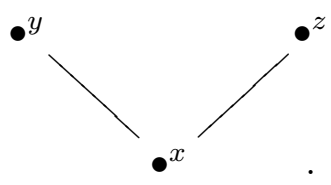

By mapping each $f \in I(X, R)$ to $\left[\begin{array}{ccc}f(x, x) & f(x, y) & f(x, z) \\ 0 & f(y, y) & 0 \\ 0 & 0 & f(z, z)\end{array}\right]$,

$I(X, R)$ is isomorphic to the $R$-algebra

$$
\left(\begin{array}{ccc}
R & R & R \\
0 & R & 0 \\
0 & 0 & R
\end{array}\right)
$$

By Theorem 3.2, $\operatorname{Soc}_{l}(I(X, R))=\left(\begin{array}{ccc}\operatorname{Soc}_{l}(R) & \operatorname{Soc}_{l}(R) & \operatorname{Soc}_{l}(R) \\ 0 & 0 & 0 \\ 0 & 0 & 0\end{array}\right)$. By Theorem 3.18, $\operatorname{Soc}_{r}(I(X, R))=\left(\begin{array}{ccc}0 & \operatorname{Soc}_{r}(R) & \operatorname{Soc}_{r}(R) \\ 0 & \operatorname{Soc}_{r}(R) & 0 \\ 0 & 0 & \operatorname{Soc}_{r}(R)\end{array}\right)$.

Hence, $\operatorname{Soc}_{l}(I(X, R)) \neq \operatorname{Soc}_{r}(I(X, R))$.

It is well-known that the left singular ideal of any ring is contained in the right annihilator of its left socle. Moreover, when the ring is left Artinian, then the left singular ideal of the ring is exactly the right annihilator of the left socle (eg. see [4, Proposition 2.1.4]). In [6, Chapter 8, p.305], it is stated that $I(X, R)$ is left Artinian if and only if $X$ is finite and $R$ is left Artinian. In view of all these results, let $R$ be an Artinian ring, thus we further provide an example such that $\operatorname{Sing}_{l}(I(X, R))=\operatorname{ann}_{r}\left(\operatorname{Soc}_{l}(I(X, R))\right)$.

By Corollary 2.8,

$$
\operatorname{Sing}_{l}(I(X, R))=I\left(X, \operatorname{Sing}_{l}(R)\right)=\left(\begin{array}{ccc}
\operatorname{Sing}_{l}(R) & \operatorname{Sing}_{l}(R) & \operatorname{Sing}_{l}(R) \\
0 & \operatorname{Sing}_{l}(R) & 0 \\
0 & 0 & \operatorname{Sing}_{l}(R)
\end{array}\right) .
$$

Note that $\operatorname{Soc}_{l}(I(X, R)) \operatorname{Sing}_{l}(I(X, R))=0$.

Let $X$ be a partially ordered set with order $\leq$, then we can create a new partially ordered set $X^{o p}=X$ with order $\leq^{o p}$ defined as

$$
x \leq y \text { if and only if } y \leq^{o p} x .
$$


This $\left(X^{o p}, \leq^{o p}\right)$ is called the opposite (dual) partially ordered set of $(X, \leq)$. Hence, $X$ and $X^{o p}$ are anti-isomorphic partially ordered sets. It is easy to verify that there is a one-to-one correspondence between left ideals of $I(X, R)$ and right ideals of $I\left(X^{o p}, R\right)$ via the map that sends $f$ to $f^{o p}$ where $f^{o p}(y, x)=f(x, y)$ for any $x, y \in X$. Hence, $\operatorname{Soc}_{l}(I(X, R)) \cong \operatorname{Soc}_{r}\left(I\left(X^{o p}, R\right)\right)$.

We call $X$ a self-dual partially ordered set, if $X$ is isomorphic to $X^{o p}$. The next proposition gives the necessary conditions for the left and the right socle of an incidence ring to be isomorphic.

Proposition 3.20. Let $R$ be any ring and $X$ be a self-dual locally finite partially ordered set with $\operatorname{Min}(X)$ is maximal antichain and $\kappa(x)$ is finite for all $x \in \operatorname{Min}(X)$. Then if $\operatorname{Soc}(R):=\operatorname{Soc}_{l}(R)=\operatorname{Soc}_{r}(R)$ then $\operatorname{Soc}_{l}(I(X, R)) \cong \operatorname{Soc}_{r}(I(X, R))$. Moreover, in this case, the left/right socle of $I(X, R)$ is

$$
\operatorname{Soc}(I(X, R))=\bigoplus_{x \in \operatorname{Min}(X)} \operatorname{Soc}(R) .
$$

Proof. Assume $\operatorname{Soc}(R):=\operatorname{Soc}_{l}(R)=\operatorname{Soc}_{r}(R)$ and $X$ is a self-dual partially ordered set. Then $\operatorname{Min}(X)$ and $\operatorname{Max}(X)$ are isomorphic, hence both are maximal antichains and $\kappa(x)$ and $\lambda(y)$ are finite for all $x \in \operatorname{Min}(X)$ and $y \in \operatorname{Max}(X)$.

By Proposition 3.2 and Proposition 3.18,

$$
\begin{gathered}
\operatorname{Soc}_{l}(I(X, R))=\bigoplus_{x \in \operatorname{Min}(X)}\{f \in I(X, \operatorname{Soc}(R)): f(u, v)=0 \text { if } u \neq x\} \\
=\bigoplus_{x \in \operatorname{Min}(X)} S(x, \operatorname{Soc}(R)) \cong \bigoplus_{y \in \operatorname{Max}(X)} T(y, \operatorname{Soc}(R)) \\
=\bigoplus_{y \in \operatorname{Max}(X)}\{f \in I(X, \operatorname{Soc}(R)): f(u, v)=0 \text { if } v \neq x\}=\operatorname{Soc}_{r}(I(X, R)) .
\end{gathered}
$$

Example 3.21. Consider the partially ordered set $X=\left\{x_{1}, x_{2}, x_{3}, x_{4}\right\}$ with the relation that $x_{1} \leq x_{k}$ and $x_{2} \leq x_{k}$ whenever $k=\{3,4\}$. The Hasse diagram of $X$ is

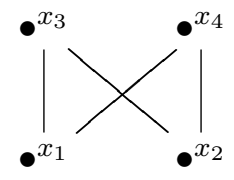

Notice that $X$ is a finite and self-dual partially ordered set, that is $X$ is isomorphic to its opposite (dual) partially ordered set, $X^{o p}$. Also $\kappa(x)$ is finite for all $x \in \operatorname{Min}(X)$. Hence the right and the left socle of $I(X, R)$ are viewed as:

$$
\begin{gathered}
\operatorname{Soc}_{l}(I(X, R))=\left(\begin{array}{cccc}
\operatorname{Soc}_{l}(R) & 0 & \operatorname{Soc}_{l}(R) & \operatorname{Soc}_{l}(R) \\
0 & \operatorname{Soc}_{l}(R) & \operatorname{Soc}_{l}(R) & \operatorname{Soc}_{l}(R) \\
0 & 0 & 0 & 0 \\
0 & 0 & 0 & 0
\end{array}\right) \\
\operatorname{Soc}_{r}(I(X, R))=\left(\begin{array}{cccc}
0 & 0 & \operatorname{Soc}_{r}(R) & \operatorname{Soc}_{r}(R) \\
0 & 0 & \operatorname{Soc}_{r}(R) & \operatorname{Soc}_{r}(R) \\
0 & 0 & \operatorname{Soc}_{r}(R) & 0 \\
0 & 0 & 0 & \operatorname{Soc}_{r}(R)
\end{array}\right) .
\end{gathered}
$$

Furthermore, when $\operatorname{Soc}_{r}(R)=\operatorname{Soc}_{l}(R)$,

$$
\operatorname{Soc}_{r}(I(X, R)) \cong \operatorname{Soc}_{l}(I(X, R)) .
$$


Remark 3.22. Note that the left and right socle of the incidence algebra are the same for the following trivial case: Assume $\operatorname{Soc}(R):=\operatorname{Soc}_{l}(R)=\operatorname{Soc}_{r}(R)$ and $X$ is an antichain. Then $I(X, R)$ is just the direct product of $|X|$-many copies of $R, I(X, R) \cong \prod_{x \in X} R$ and the socle of $I(X, R)$ is $\bigoplus_{x \in X} \operatorname{Soc}(R)$.

Another example is when $\operatorname{Min}(X)=\emptyset$. Consider the case $X=\mathbb{Z}$. Since $\operatorname{Min}(X)=\emptyset$ by Lemma 3.4, $\operatorname{Soc}_{l}(I(X, R))=\{0\}$ and analogously $\operatorname{Max}(X)=\emptyset, \operatorname{Soc}_{r}(I(X, R))=\{0\}$.

Acknowledgment. This research question has been motivated by an idea of Prof. Eugene Spiegel of University of Connecticut while the first author was conducting her Ph.D. under his supervision. This long time overdue paper is dedicated to his memory. The authors are deeply thankful to Nesin Math Village, Şirince, Izmir for providing an excellent research environment where this work was completed, to Noyan Er for fruitful conversations and to the referees on a detailed report and their helpful comments that improved the paper and Proposition 3.20.

\section{References}

[1] F. Al-Thukair, S. Singh and I. Zaguia, Maximal ring of quotients of an incidence algebra, Arch. Math. 80, 358-362, 2003.

[2] S. Esin, M. Kanuni and A. Koç, Characterization of some ring properties in incidence algebras, Comm. Algebra, 39 (10), 3836-3848, 2011.

[3] M. Kanuni, Dense ideals and maximal quotient rings of incidence algebras, Comm. Algebra, 31 (11), 5287-5304, 2003.

[4] T.Y. Lam, Lectures on Modules and Rings, Graduate Texts in Mathematics 189, New York-Berlin, Springer-Verlag, 1999.

[5] E. Spiegel, Essential ideals of incidence algebras, J. Austral. Math. Soc. (Series A), 68, 252-260, 2000.

[6] E. Spiegel and C.J. O'Donnell, Incidence Algebras, Monographs and Textbooks in Pure Appl. Math. 206, New York, Marcel Dekker, 1997. 